\title{
Should community health workers offer support healthcare services to survivors of sexual violence? a systematic review
}

\author{
Anne Gatuguta ${ }^{1,2^{*}}$, Barbra Katusiime ${ }^{3,4}$, Janet Seeley ${ }^{1}$, Manuela Colombini ${ }^{1}$, Isaac Mwanzo ${ }^{2}$ and Karen Devries ${ }^{1}$
}

\begin{abstract}
Background: Sexual violence is widespread, yet relatively few survivors receive healthcare or complete treatment. In low and middle-income countries, community health workers (CHWs) have the potential to provide support services to large numbers of survivors. The aim of this review was to document the role of CHWs in sexual violence services. We aimed to: 1) describe existing models of CHWs services including characteristics of CHWs, services delivered and populations served; 2) explore acceptability of CHWs' services to survivors and feasibility of delivering such services; and 3) document the benefits and challenges of CHW-provided sexual violence services.

Methods: Quantitative and qualitative studies reporting on CHWs and other community-level paraprofessional volunteer services for sexual violence were eligible for inclusion. CHWs and sexual violence were defined according to WHO criteria. The review was conducted according to the Preferred Reporting Items for Systematic reviews and Meta-Analyses guidelines. Quality of included studies was assessed using two quality assessment tools for quantitative, and, the methodology checklist by the National Institute for Health and Clinical Excellence for qualitative studies. Data were extracted and analysed separately for quantitative and qualitative studies and results integrated using a framework approach.
\end{abstract}

Results: Seven studies conducted in six countries (Democratic Republic of Congo, Rwanda, Burma, United States of America, Scotland, Israel) met the inclusion criteria. Different models of care had diverse CHWs roles including awareness creation, identifying, educating and building relationships with survivors, psychosocial support and follow up. Although sociocultural factors may influence CHWs' performance and willingness of survivors to use their services, studies often did not report on CHWs characteristics. Few studies assessed acceptability of CHWs' to survivors or feasibility of delivery of services. However, participants mentioned a range of benefits including decreased incidence of violence, CHWs being trusted, approachable, non-judgmental and compassionate. Challenges identified were high workload, confidentiality issues and community norms influencing performance.

Conclusions: There is a dearth of research on CHWs services for sexual violence. Findings suggest that involving CHWs may be beneficial, but potential challenges and harms related to CHW-provided services exist. No different models of CHW-provided care have been robustly evaluated for effects on patient outcomes. Further research to establish survivors' views on these services, and, their effectiveness is desperately needed.

Keywords: Sexual violence, Community health workers, Sexual violence healthcare services, Survivors

\footnotetext{
* Correspondence: Anne.Gatuguta@lshtm.ac.uk

${ }^{1}$ Department of Global Health and Development, Faculty of Public Health

and Policy, London School of Hygiene and Tropical Medicine, Keppel Street,

London WC1E 7HT, UK

${ }^{2}$ Department of Community Health, School of Public Health, Kenyatta

University, Nairobi, Kenya

Full list of author information is available at the end of the article
}

(c) The Author(s). 2017 Open Access This article is distributed under the terms of the Creative Commons Attribution 4.0 International License (http://creativecommons.org/licenses/by/4.0/), which permits unrestricted use, distribution, and reproduction in any medium, provided you give appropriate credit to the original author(s) and the source, provide a link to the Creative Commons license, and indicate if changes were made. The Creative Commons Public Domain Dedication waiver (http://creativecommons.org/publicdomain/zero/1.0/) applies to the data made available in this article, unless otherwise stated. 


\section{Background}

Sexual violence is widespread globally. Reported lifetime prevalence of partner and non-partner sexual violence in women is as high as $59 \%$ and $12 \%$ respectively in some regions [1-3]. Associated health consequences are both short-term and long-term [1, 4-10]. Global clinical guidelines recommend comprehensive immediate healthcare and follow up to address both the clinical and psychosocial needs of survivors [11-15]. However, the majority of survivors do not access health care and only a limited proportion complete recommended treatment [16-25]. Sexual violence stigma at the community level, distance from health facilities, unreliable or unavailable services, healthcare professionals' attitudes and competing priorities for survivors such as work are some of the main barriers to access [25-27]. Lack of active follow up and social support further hinder treatment completion [18, 25]. Moreover, most services are provided through emergency care or rape care centres, where fear of stigma and being judged may prevent participants from attending [18]. One way to overcome the poor access to healthcare and to treatment completion is to make use of already existing and affordable structures such as community health worker (CHW) services [28, 29].

The World Health Organisation (WHO) defines CHWs as community-based workers who are members of the communities where they work, selected by their communities, have received limited training but are not professional health workers. They are supported by the health system while not necessarily being a part of its organisation [30]. Community health workers provide a means for communities to access affordable healthcare as well as participate in managing their health [31]. Studies show that CHWs can provide benefits in cost-savings, increasing community involvement, improving clinical outcomes, providing an alternative to professional workforce-limited situations and decongesting health facilities [32].

There have been concerted efforts, particularly in resource-limited settings, to utilise $\mathrm{CHWs}$ in the management of different health conditions. Data show the positive impact of $\mathrm{CHWs}$ on access to care, clinical, retention and other outcomes in treatment of specific health conditions such as maternal and child health [33], tuberculosis (TB) [34], human immunodeficiency virus/ acquired immune deficiency syndrome (HIV/AIDS) [32, $34,35]$ and mental health [36, 37]. A systematic review of community-based HIV treatment in Sub-Saharan Africa for instance, showed that interventions delivered by CHWs could reduce barriers to retention and reduce costs to patients [38].

In this paper we report the findings of a review to investigate whether $\mathrm{CHWs}$ could provide similar benefits in sexual violence healthcare for adults and children. We reviewed different components of $\mathrm{CHW}$ programmes that could potentially influence $\mathrm{CHW}$ performance as well as interactions with survivors. These components included the socio-demographic characteristics of CHWs used, their selection, training provided, roles of the CHWs, mode of service delivery and population served. The review also assessed the acceptability of CHWs services by survivors, the feasibility of delivering such services, as well as the challenges and benefits associated with such services for $\mathrm{CHWs}$, health care systems and survivors.

\section{Methods}

We conducted a systematic review of qualitative and quantitative studies. The review was conducted and reported according to the Preferred Reporting Items for Systematic reviews and Meta-Analyses (PRISMA) guidelines [39] and was registered with the International Prospective Register of Systematic Reviews (PROSPERO).

Inclusion criteria:

All studies that reported on services or interventions delivered by CHWs to support prevention of sexual violence, access to healthcare, treatment adherence and retention in healthcare for sexual violence survivors were included. Community health workers were defined based on the WHO definition [30]. Many of the studies referred to these workers as volunteers and therefore the term CHWs and volunteers are used here interchangeably. Sexual violence was defined based on the WHO definition as "any sexual act, attempt to obtain a sexual act, unwanted sexual comments or advances, or acts to traffic, or otherwise directed, against a person's sexuality using coercion, by any person regardless of their relationship to the victim, in any setting, including but not limited to home and work" [40].

\section{Search strategy}

Seven databases were searched from first record to $17 /$ 05/2017: MEDLINE, Africa Wide Information, Cumulative Index to Nursing and Allied Health Literature (CINAHL) Plus, Cochrane library, Embase, Global Health and PsychINFO. References of identified studies were also checked for relevant studies. There was no time or language restriction to the studies. A search criteria tailored for each database was developed with the relevant controlled vocabulary terms, Boolean operators and truncation applied to the different databases. The search terms for CHWs included community health worker*, $\mathrm{CHW}^{*}$, lay health worker*, community own resource person*, CORP*, patient advocate ${ }^{*}$, close to community health worker*, community health aide*, and village health worker. The search terms for sexual violence included sexual violence, sexual abuse, sexual molestation, sexual assault, rape, date rape, defilement, incest, sodomy, child sexual abuse, post exposure 
prophylaxis, PEP, ARV*, antiretroviral*, HIV PEP, nPEP and n-PEP. The search strategy and number of articles obtained is outlined in Additional file 1: Appendix 1.

In total, 4617 records were obtained, saved into an EndNote X7 library and duplicates removed. The remaining 3901 records were screened on titles and abstracts and irrelevant studies identified excluded from further screening. Full texts were obtained for 24 studies. A further 17 studies were excluded based on the full text review due to various reasons as shown (Fig. 1). All the titles and abstracts for inclusion were screened by AG; AG and BK read and screened the 24 full text studies.

\section{Data abstraction}

All information related to supporting prevention, access to care, treatment and retention was extracted. Acceptability and feasibility were assessed through reported willingness to use, satisfaction with services, ease of delivery, quality and uptake of services, availability of resources, adequacy of training and costeffectiveness of services. Additionally, data extracted included: the type of study design, study setting, participants socio-demographic characteristics, number of participants, study methods including recruitment and retention, outcomes measured and results.
For qualitative studies, all data and themes identified by the author in the paper were abstracted [41]. As our study is exploratory, we took this more inclusive approach to allow more data and themes to be included for analysis [41]. Data were abstracted on data collection methods used including number and composition of group members, consenting process, data processing and analysis, identified themes and findings from the study.

\section{Methodological quality of included studies}

Quantitative and qualitative studies were assessed separately for methodological quality. The quality of the studies was considered critically and findings are interpreted in light of this, however, no studies were excluded based on their quality. As the two quantitative studies were vastly different in design (one a longitudinal descriptive study and the other a pre and posttest design) two quality assessment tools were used. Quality assessment tool for quantitative studies developed by the Effective Public Health Practice Project (EPHPP) was used for the pre and post-test study [42] while quality assessment checklist for observational studies (QATSO Score) was used for the longitudinal study [43]. An overall rating of the whole study is

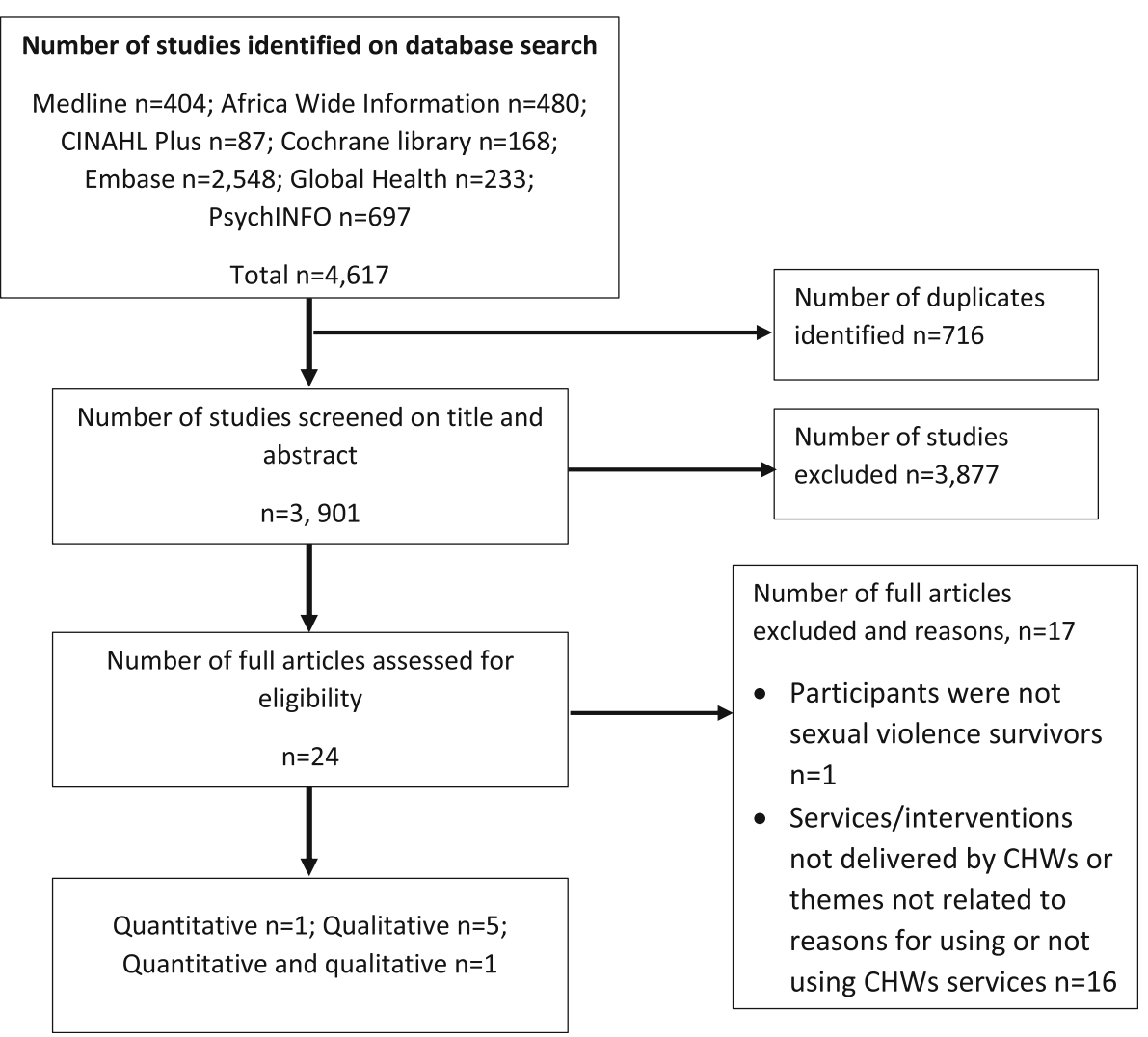

Fig. 1 Literature search results 
normally constituted from all quality domains; however the Cochrane collaboration discourages the assigning of a summary score as this involves assigning weights to different domains which may not be justifiable [44]. For this reason, both quantitative studies were rated on each domain but an overall quality score was not constituted (Additional file 1: Appendix 2 ). Quality assessment for the qualitative studies was done using the methodology checklist for qualitative studies by the National Institute for Health and Clinical Excellence (NICE) [45] and is shown in Additional file 1: Appendix 3.

\section{Bias}

Due to limited time and resources, a comprehensive search for grey literature was not done, so some studies may not have been included. Nevertheless, publication bias was minimised by searching multiple databases as reported, and although not comprehensively done, Google Scholar, the London School of Hygiene and Tropical Medicine Research Online and the WHO's website were also searched. Too few studies using the same outcome measures met the inclusion criteria to quantitatively assess publication bias. We did not find multiple articles reporting on the same study. No studies were excluded based on language and no time period was applied to the search.

\section{Data analysis and synthesis}

Qualitative and quantitative data were analysed separately and the findings combined into a final synthesis. The two quantitative studies reviewed had different aims and outcomes, therefore a descriptive synthesis was performed in lieu of a meta-analysis. For qualitative studies, data were analysed thematically. Key themes relevant to the review questions were identified prior to data abstraction. Other relevant themes emerging from the review process were integrated in the analysis.

Data synthesis from both qualitative and quantitative studies was done through a framework approach [46] and summarised in matrices as illustrated in Table 1. The findings were summarised under the following headings: models of $\mathrm{CHW}$ services for sexual violence care, acceptability and feasibility of $\mathrm{CHW}$ services in sexual violence care and the challenges and benefits of $\mathrm{CHWs}$ as service providers for sexual violence.

\section{Results}

\section{Characteristics of included studies}

Seven studies met the inclusion criteria [47-53]. The studies were conducted in six countries: Democratic Republic of Congo, Rwanda, Burma, United States of America, Scotland and Israel. Of those included, five were qualitative [48, 50-53], one quantitative [49] and one used both quantitative and qualitative methods [47]. Of the five qualitative studies, one was implemented for prevention of child sexual abuse in the community [48], the second described a volunteer advocate support programme for a specific population of the deaf and deaf-blind [50]. The third study described a stand-alone treatment centre for sexual violence which utilised volunteers to provide psychosocial support to survivors [51]. The fourth study described a pilot programme for community-based medical care for survivors delivered by CHWs [52] while the fifth comprised of 44 semistructured interviews with survivors who were members of local survivors associations where select members were trained to provide trauma counselling to their peers [53].

The quantitative study was a longitudinal descriptive study that followed up survivors over a one month period and utilized CHWs attached to a mobile clinic at the community level [49]. The mixed methods study involved a pre- and post-test waitlist design with a volunteer-delivered prevention programme. Quantitative methods were used to compare pre- and post-intervention knowledge, skills and occurrence of violence disclosure while qualitative methods were used to assess acceptability and feasibility of the programme [47]. The characteristics of the studies are summarised in Table 2.

\section{Methodological quality of quantitative studies}

Only two quantitative studies were identified which met our inclusion criteria. One was a longitudinal descriptive study of survivors receiving medical treatment [49]; the other was a small pilot test of a prevention intervention with only 20 participants, which used a pre and post-test design [47]. The quality of the studies is summarised in Table 3. These are some of the first studies conducted on this topic and provide valuable information about how participants interact with services. However, the sample size was very small in the pre-post study, and attrition was extremely high in the longitudinal study (perhaps not surprisingly given that it was conducted in a conflict setting). No studies were found that intended to evaluate the effects of a $\mathrm{CHW}$ led intervention on survivor outcomes.

\section{Methodological quality of qualitative studies}

Three of the qualitative studies were scored as having met most of the checklist criteria $(++)[47,52,53]$, one was scored as having met some of the criteria $(+)$ [48] and two were scored as having met few of the criteria $(-)[50,51]$. The quality assessment was limited by a lack of detail about reported methods, particularly in regards to data collection and analysis. The quality assessment for all the qualitative studies is summarised in Table 4. 


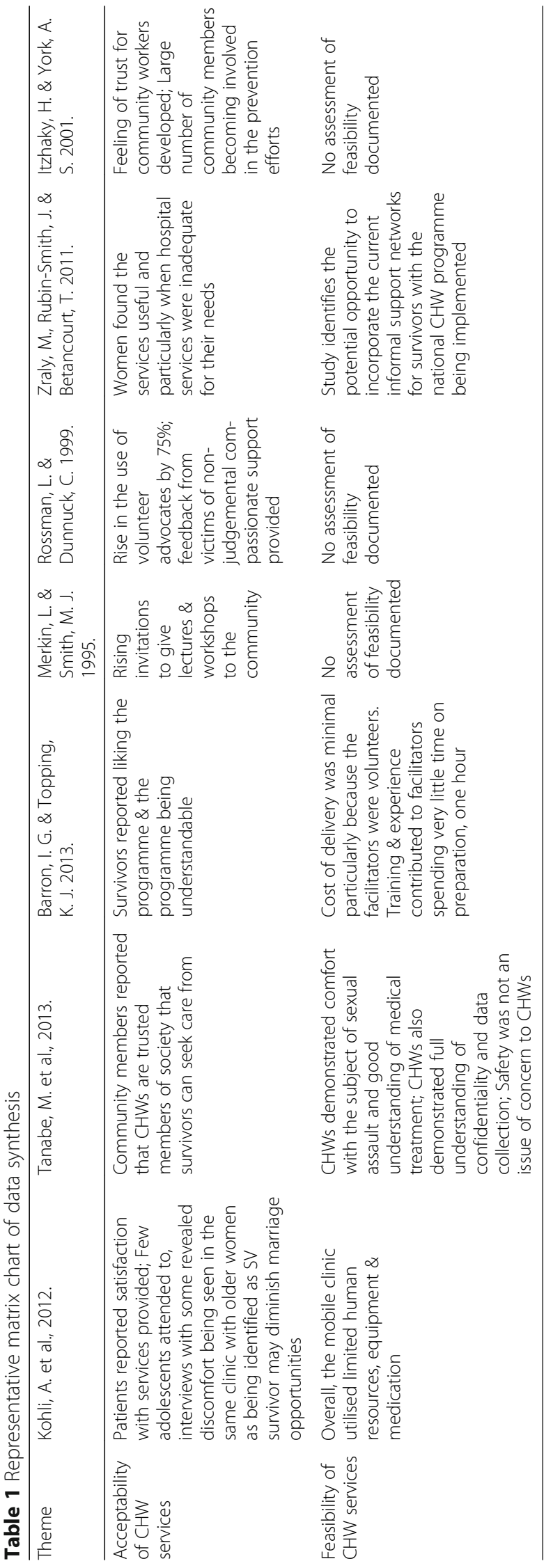




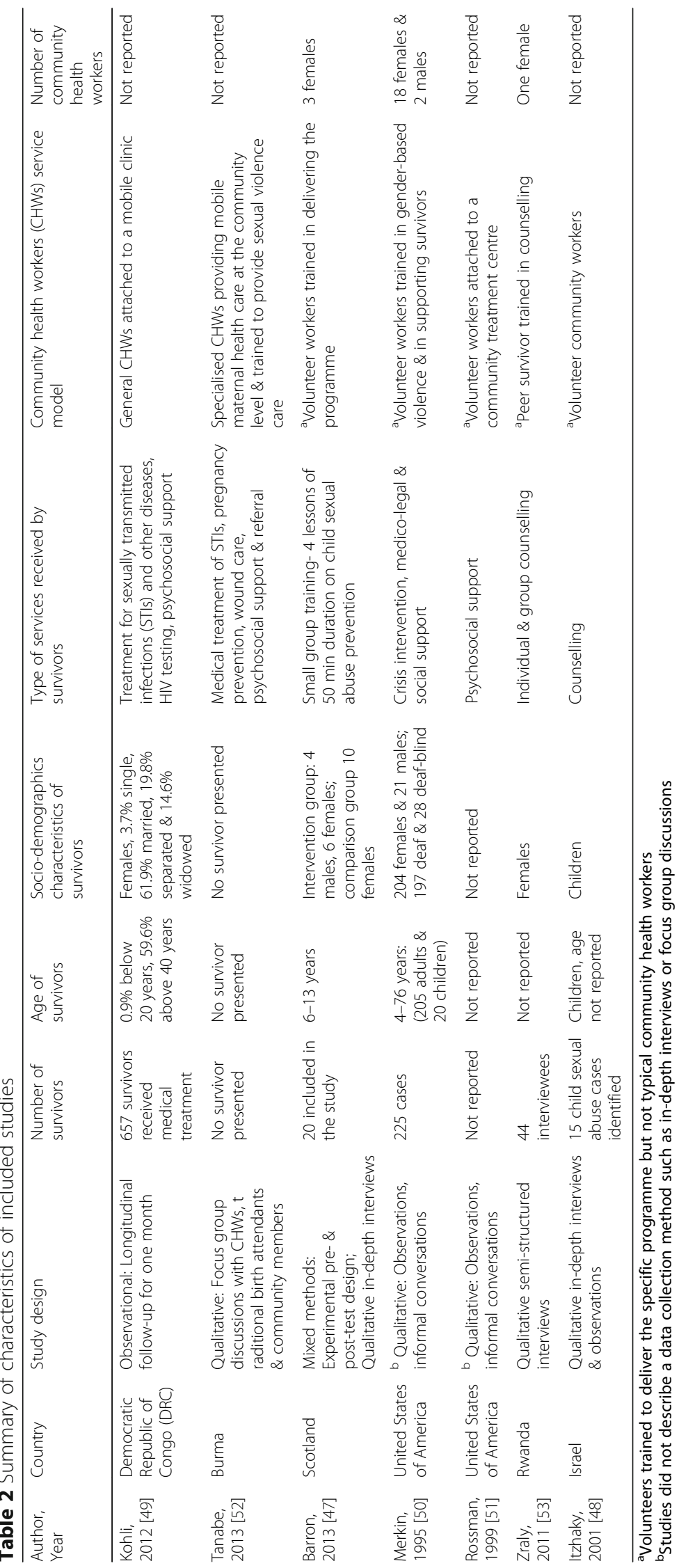


Table 3 Methodological quality rating of quantitative studies

\begin{tabular}{|c|c|c|c|c|c|c|c|c|}
\hline \multirow[t]{2}{*}{ Study } & \multicolumn{8}{|c|}{ Rating domain (EPHPP for evaluation studies) } \\
\hline & $\begin{array}{l}\text { Selection } \\
\text { bias }\end{array}$ & $\begin{array}{l}\text { Study } \\
\text { design }\end{array}$ & Confounders & Blinding & $\begin{array}{l}\text { Data collection } \\
\text { methods }\end{array}$ & $\begin{array}{l}\text { Withdrawals and } \\
\text { dropouts }\end{array}$ & $\begin{array}{l}\text { Intervention } \\
\text { integrity }\end{array}$ & Analyses \\
\hline \multirow{3}{*}{$\begin{array}{l}\text { Barron, } 2013 \\
\text { [47] }\end{array}$} & Moderate & Moderate & Weak & Not applicable & Moderate & Strong & Moderate & Moderate \\
\hline & \multicolumn{8}{|c|}{ Rating domain (QUATSO for) } \\
\hline & External validity & & Reporting & & Confounding & & Bias (Privacy) & \\
\hline \multirow[t]{2}{*}{ Kohli, 2012 [49] } & 0 & & $\begin{array}{l}\text { Response } \\
\text { rate }\end{array}$ & $\begin{array}{l}\text { Outcome } \\
\text { measure }\end{array}$ & Not applicable & & 1 & \\
\hline & & & $\begin{array}{l}\text { Not } \\
\text { applicable }\end{array}$ & 1 & & & & \\
\hline
\end{tabular}

\section{Components of $\mathrm{CHW}$ models for sexual violence services} The components of $\mathrm{CHW}$ models reported include the socio-demographic characteristics of $\mathrm{CHWs}$, their selection, training, roles, mode of service delivery and population served (Table 5). In general, $\mathrm{CHW}$ services were delivered by volunteers trained on the specific intervention. Most were specialised volunteers who only delivered one type of service/intervention and there was no evidence that they carried out any other health-related activities [47, 50, 51, 53]. In nearly all cases, the volunteer programmes were run by nongovernmental organisations (NGOs) but delivered services in collaboration with government agencies.

\section{Socio-demographic characteristics of CHWs used}

Only 3 of 7 studies reported any socio-demographic characteristics of the CHWs. One study reported using only female CHWs aged between 20 and 30 years [47] while another reported using both females and gay men to serve gay male survivors [50]. A third study described using peers who were female [53]. Even among the studies that reported this information, none reported how these characteristics may or may not have affected the outcomes measured or relationships with survivors.

\section{Selection of CHWs}

The selection of the CHWs was reported in 3 of 7 studies. Where reported, the selection appeared to target CHWs with specific skills suited to serving survivors. One study reported selecting the 'highest cadre' of CHWs, defined as CHWs who had previously been trained to provide reproductive health services [52]. These CHWs were therefore deemed already equipped with the skills they needed, for instance to provide emergency contraception to survivors.

Table 4 Methodological quality assessment for qualitative studies

\begin{tabular}{|c|c|c|c|c|c|c|}
\hline Rating section & $\begin{array}{l}\text { Barron, } 2013 \\
{[47]}\end{array}$ & $\begin{array}{l}\text { Itzhaky, } 2001 \\
{[48]}\end{array}$ & $\begin{array}{l}\text { Merkin, } 1995 \\
{[50]}\end{array}$ & $\begin{array}{l}\text { Rossman, } 1999 \\
{[51]}\end{array}$ & $\begin{array}{l}\text { Tanabe, } 2013 \\
{[52]}\end{array}$ & $\begin{array}{l}\text { Zraly, } 2011 \\
{[53]}\end{array}$ \\
\hline 1.1 Is a qualitative approach appropriate? & Appropriate & Appropriate & Appropriate & Appropriate & Appropriate & Appropriate \\
\hline 1.2 Is the study clear in what it seeks to do? & Clear & Mixed & Mixed & Unclear & Clear & Mixed \\
\hline $\begin{array}{l}2.1 \text { How defensible/rigorous is the research } \\
\text { design/methodology? }\end{array}$ & Defensible & Defensible & Not defensible & Not defensible & Defensible & Defensible \\
\hline $\begin{array}{l}3.1 \text { How well was the data collection } \\
\text { carried out? }\end{array}$ & Appropriate & Appropriate & $\begin{array}{l}\text { Inadequately } \\
\text { reported }\end{array}$ & $\begin{array}{l}\text { Inadequately } \\
\text { reported }\end{array}$ & Appropriate & Appropriate \\
\hline 4.1 Is the context clearly described? & Clear & Clear & Unclear & Unclear & Clear & Clear \\
\hline 4.2 Were the methods reliable? & Reliable & Reliable & Unreliable & Unreliable & Unreliable & Unreliable \\
\hline 5.1 Are the data 'rich'? & Rich & $\begin{array}{l}\text { Not sure/not } \\
\text { reported }\end{array}$ & Not reported & Not reported & Rich & Rich \\
\hline 5.2 Is the analysis reliable? & Reliable & Not reported & Not reported & Not reported & Unreliable & Reliable \\
\hline 5.3 Are the findings convincing? & Convincing & Not convincing & Not convincing & Convincing & Convincing & Convincing \\
\hline 5.4 Are the conclusions adequate? & Adequate & Adequate & Adequate & Adequate & Adequate & Adequate \\
\hline $\begin{array}{l}\text { 6.1 Was the study approved by an ethics } \\
\text { committee? }\end{array}$ & Yes & Not reported & Not reported & Not reported & Yes & Yes \\
\hline $\begin{array}{l}6.2 \text { Is the role of the researcher clearly } \\
\text { described? }\end{array}$ & Clear & Not clear & Not clear & Not reported & Clear & Clear \\
\hline $\begin{array}{l}\text { As far as can be ascertained from the paper, } \\
\text { how well was the study conducted? }\end{array}$ & ++ & + & - & - & ++ & ++ \\
\hline
\end{tabular}




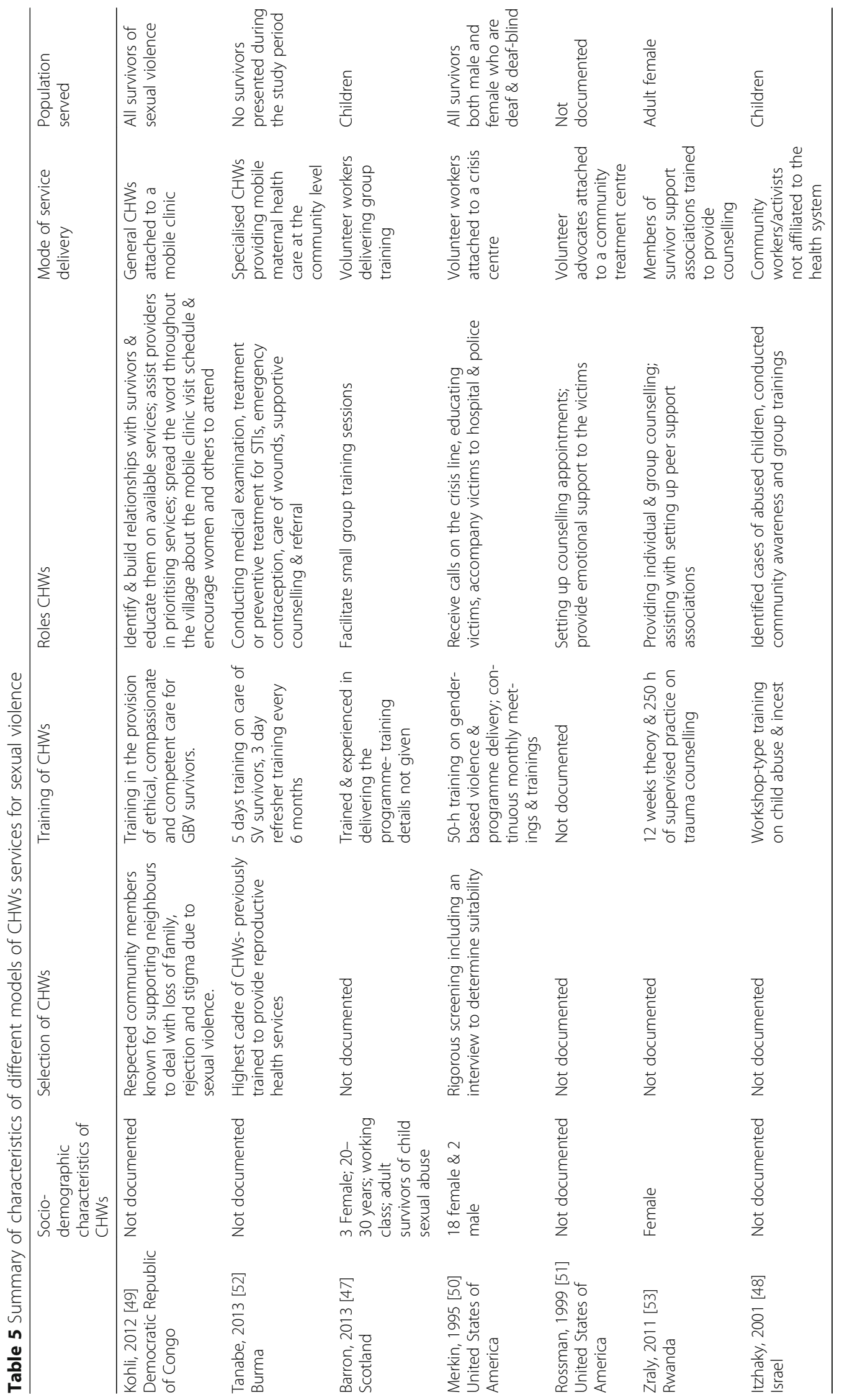


A second study reported screening volunteers for their ability to understand the importance of confidentiality and sensitivity [50]. A third study described selecting respected community members already known for supporting individuals dealing with grief, rejection and sexual violence stigma [49].

\section{Training of CHWs}

The training details for the CHWs were not given in two studies $[47,51]$. In the other five studies, the duration of training varied broadly, and there was a lack of detail about the content of training curricula. One study reported a 'workshop type' training on child abuse and incest [48]. The training materials included topics on child abuse, dynamics of abusing families, societal attitudes towards child sexual abuse (CSA), how to communicate with victims and reporting of abuse among others. Two studies reported at least five days training with frequent refresher courses $[50,52]$. The topics covered included medical care of survivors, psychosocial support and referral. The fourth study reported 12 weeks of theory and $250 \mathrm{~h}$ of practice on trauma counselling [53] while the fifth only mentioned providing training in the provision of ethical, compassionate and competent care for gender-based violence survivors [49]. None of the studies assessed the effect, duration or the contents of training on any outcomes related to survivors or CHWs.

\section{Roles of the CHWs and mode of service delivery}

Different modes of service delivery were reported. In some programmes, CHWs worked at the community level $[48,52,53]$ while in others they were based in a facility $[50,51]$ and yet in others they were involved both at the community and facility [49]. For those based in the community, activities included raising awareness, identifying cases, treatment, providing community feedback to healthcare workers at health facilities and providing psychosocial support including individual and group counselling of survivors. Those based in facilities responded to crisis telephone calls, accompanied survivors to hospitals and the police, provided emotional support and education as well as assisted clinicians in tasks related to managing survivors such as prioritising treatment, setting up appointments and follow-up. One programme had volunteers providing group-training on knowledge and skills for prevention of child sexual abuse to children with previous exposure to violence. The role of treating survivors including wound care, prescribing treatment such as emergency contraception and prophylaxis for sexually transmitted infections was piloted by one study [52]. Although this pilot was carried out for one year and CHWs reported being comfortable providing these services, no survivor was treated and it is therefore difficult to draw any conclusions on the capacity of CHWs delivering medical care to survivors.

\section{Populations served}

With regards to populations served, the prevention services targeted prevention of violence in children with interventions delivered to both the community and groups of children [47, 48]. Services and interventions providing medical care and psychosocial support targeted adult women $[49,52]$. In some instances, the services were provided as part of a support group formed by survivors and ran by peers [53]. One programme was designed exclusively for a specific population of the deaf and deafblind which included both males and females [50]. It is notable however, that the proportion of clients who were males served by this intervention was low (9.3\%).

\section{Acceptability and feasibility of CHWs services in sexual violence care}

Assessment of the acceptability and feasibility of CHW services was limited and equivocal as summarised in Table 6. Only three studies reported collecting data from survivors on their experience with CHWs services [47, 51, 53]. Survivors in one study found the services useful in improving access to care and providing psychosocial support particularly when hospital services were inadequate for their needs [53]. In the other two studies, programmes delivered by $\mathrm{CHWs}$ were reported as likeable and understandable as well as providing nonjudgemental and compassionate support $[47,51]$. These findings on acceptability are limited by the small number of survivors interviewed. It is also notable that the studies either did not assess or did not report any negative concerns from survivors.

Notably, only one of the studies in the review was designed specifically to assess the performance of CHWs in delivering healthcare services to survivors [52]. In this study, community members interviewed expressed the view that CHWs were trusted members of the community who could be approached by survivors for help. Although the study design included a plan to interview survivors, this did not happen as no survivor presented within the one year study period. The reasons why survivors did not present are not clear, with the authors suggesting possible reasons as no incidence occurring, sensitivity of the subject or lack of awareness of the existence of services. The other studies were designed to assess programmes in which CHWs were a component. As such, reported data focused more on the overall programme acceptability rather than the CHWs. Additionally, reports are mainly from other stakeholders' perspectives and not the survivors themselves thus posing an impediment to the extent that acceptability of the services could be assessed. 
Table 6 Reported acceptability and feasibility of CHWs in sexual violence services

\begin{tabular}{|c|c|c|}
\hline & Acceptability & Feasibility \\
\hline Kohli, 2012 [49] & $\begin{array}{l}\text { Not documented- assessment of whole programme rather } \\
\text { than CHWs }\end{array}$ & $\begin{array}{l}\text { Not documented-assessment of whole programme rather } \\
\text { than CHWs }\end{array}$ \\
\hline Tanabe, 2013 [52] & $\begin{array}{l}\text { Community members interviewed reported that CHWs are } \\
\text { trusted members of society that survivors can seek care } \\
\text { from }\end{array}$ & $\begin{array}{l}\text { CHWs demonstrated comfort with the subject of sexual } \\
\text { assault and good understanding of medical treatment; } \\
\text { CHWs demonstrated full understanding of confidentiality } \\
\text { and data collection; Safety was not an issue of excess } \\
\text { concern to CHWs }\end{array}$ \\
\hline Barron, 2013 [47] & $\begin{array}{l}\text { Survivors reported liking the programme \& the } \\
\text { programme being understandable }\end{array}$ & $\begin{array}{l}\text { Cost of delivery was minimal particularly because the } \\
\text { facilitators were volunteers. Training \& experience } \\
\text { contributed to facilitators spending very little time on } \\
\text { preparation }\end{array}$ \\
\hline Merkin, 1995 [50] & $\begin{array}{l}\text { Not documented- assessment of programme rather than } \\
\text { CHWs }\end{array}$ & No assessment of feasibility documented \\
\hline Rossman, 1999 [51] & $\begin{array}{l}\text { Rise in the use of volunteer advocates by } 75 \% \text {; feedback } \\
\text { from victims of non-judgemental compassionate support } \\
\text { provided }\end{array}$ & No assessment of feasibility documented \\
\hline Zraly, 2011 [53] & $\begin{array}{l}\text { Interviewed women found the services useful and } \\
\text { particularly when hospital services were inadequate for } \\
\text { their needs }\end{array}$ & No assessment of feasibility documented \\
\hline Itzhaky, 2001 [48] & $\begin{array}{l}\text { Feeling of trust for community workers developed; Large } \\
\text { number of community members becoming involved in } \\
\text { the prevention efforts }\end{array}$ & No assessment of feasibility documented \\
\hline
\end{tabular}

Only two studies assessed any aspect of feasibility of sexual violence service delivery by CHWs. Interviewed CHWs reported being comfortable dealing with sexual violence, understanding the services they were to provide and the confidentiality required, and having no safety concerns [52]. The second study assessed the cost of delivering the programme and reported it to be minimal due to $\mathrm{CHW}$ s being volunteers therefore unpaid [47]. However, this was not a formal costing but an estimate of the cash used to deliver the programme. This study also reported limited preparation time for the CHWs once they had received initial training and gained experience.

\section{Challenges and benefits of $\mathrm{CHWs}$ providing services for sexual violence}

Several benefits and challenges were mentioned (Table 7). Authors in two studies reported that enhanced community awareness and knowledge as a result of CHWs activities and advocacy resulted in fewer incidences of violence, increased number of survivors taking action to end violence from partners and an increased number of cases of abuse going to trial $[48,50]$. Nevertheless, these studies were small and larger prospective studies with control groups are needed to assess the effect of similar interventions over time. Two studies reported feedback from survivors that they received non-judgemental, compassionate and useful psychosocial support from CHWs $[51,53]$. Similarly, the authors of one study observed that the programme benefited by having CHWs among their cadre of staff [49]. The CHWs provided feedback that assisted healthcare providers to understand the local concerns which they could then address during health talks or individual treatment. They were also able to mobilise communities and survivors to access the available healthcare which they might have missed without the awareness created.

There were general challenges related to CHWs providing sexual violence services and challenges related to specific models of CHWs. Where CHWs provided other health-related services (general CHWs), issues of many responsibilities and high workload were reported $[49,52]$. In addition, other commitments that CHWs had, prevented them from delivering the services effectively and in some instances, CHWs were expected to cover vast regions hindering their ability to reach everyone [49].

With regards to sexual violence, some of the CHWs interviewed were uncomfortable with certain aspects of care, (for example, medical care) while others were unclear on how to assist survivors while maintaining confidentiality [52]. In one study, professional healthcare workers and survivors had a problem understanding the role of the CHWs which meant they were not always readily accepted as part of the care team [51]. Additionally, in programmes where CHWs were called in when a survivor presented, the time taken to contact the CHWs and get them to the facility was often long, therefore delaying care for the survivor [51]. One study reported concerns regarding community norms affecting the work of the CHWs who were themselves members of that community [48]. Child abuse was noted as being normative in 
Table 7 Reported benefits and challenges of CHWs in sexual violence services

\begin{tabular}{|c|c|c|}
\hline & Benefits & Challenges \\
\hline Kohli, 2012 [49] & $\begin{array}{l}\text { Authors report that local CHWs assisted healthcare } \\
\text { providers in targeting education sessions to community } \\
\text { concerns; CHWs provided feedback to healthcare providers } \\
\text { e.g. reported increased patient satisfaction }\end{array}$ & $\begin{array}{l}\text { Authors report "travel distance \& other commitments } \\
\text { sometimes prevented CHWs from reminding patients } \\
\text { about appointments and thus, follow-up rates were not as } \\
\text { high as expected." }\end{array}$ \\
\hline Tanabe, 2013 [52] & $\begin{array}{l}\text { Community reported that } \mathrm{CHW} \text { s are trusted persons that } \\
\text { survivors can approach for help }\end{array}$ & $\begin{array}{l}\text { CHWs reported lacking confidence in history-taking and } \\
\text { psychosocial care; 'Lower cadres' of CHWs were unhappy } \\
\text { with some aspects of medical care \& referrals, complained } \\
\text { they already had too many responsibilities, had issues with } \\
\text { maintaining confidentiality \& had some safety concerns }\end{array}$ \\
\hline Barron, 2013 [47] & $\begin{array}{l}\text { Increased knowledge \& skill; Occurrence of disclosures in } \\
\text { the intervention group compared to no disclosures in the } \\
\text { comparison group; satisfaction with programme; minimal } \\
\text { cost of delivery }\end{array}$ & Not documented \\
\hline Merkin, 1995 [50] & $\begin{array}{l}\text { Increased number of victims taking action on violence in } \\
\text { their lives \& increase in number of cases of abuse going to } \\
\text { trial }\end{array}$ & Not documented \\
\hline Rossman, 1999 [51] & $\begin{array}{l}\text { Feedback from victims report non-judgemental compas- } \\
\text { sionate support by volunteers }\end{array}$ & $\begin{array}{l}\text { Time taken to contact the volunteer \& get them to the } \\
\text { centre to offer support was long delaying care for } \\
\text { survivors; Failure of recognition \& acceptance by both the } \\
\text { victim \& professional healthcare workers }\end{array}$ \\
\hline Zraly, 2011 [53] & Available care in crisis \& source of support & Not documented \\
\hline Itzhaky, 2001 [48] & $\begin{array}{l}\text { Increased community awareness with change of attitude } \\
\text { towards child sexual abuse; Reduction in stigma \& } \\
\text { therefore increased acceptance \& support for survivors; } \\
\text { Reduced incidence of cases }\end{array}$ & $\begin{array}{l}\text { Child abuse reportedly normative thus community workers } \\
\text { not motivated to act initially }\end{array}$ \\
\hline
\end{tabular}

this community and the CHWs also had difficulties recognising it as abuse.

\section{Discussion}

To our knowledge, this is the first systematic review of $\mathrm{CHW}$ services for sexual violence against adults and children. This review has identified important gaps in research in this area. Firstly, there was no robust evidence to support any particular model or model components as being effective for the delivery of sexual violence services by CHWs. Secondly, evidence on the acceptability and feasibility of delivering these services is minimal in volume and generally limited in quality. Although there is some evidence suggesting that CHWs provide services for survivors, the experiences of the survivors themselves and CHWs with regards to these services are largely undocumented. Thirdly, in terms of benefits, the studies reviewed indicate a wide range of services provided by CHWs spanning from prevention, treatment, psychosocial support and follow up. However, it is unclear where $\mathrm{CHW}$ s provide the maximum benefit or where their knowledge and skills can be optimally utilised. Finally, various challenges were mentioned which highlight the importance of tailoring services to survivor needs, different populations and context and this area requires further exploration.

Our review had several limitations. Firstly, there were limitations related to the included studies: Few studies met the inclusion criteria. Very few survivors or CHWs were interviewed in these studies, therefore the studies mostly represent the views of other stakeholders rather than the actual consumers of services. It is therefore difficult to draw any conclusions on effectiveness of models, acceptability or feasibility of CHWs in sexual violence services. No studies evaluated the effects of any $\mathrm{CHW}$-provided intervention to improve any aspect of sexual violence care. These limitations perhaps reflect the difficulties of conducting research in this topic and highlight the need for innovative ways to recruit and follow up survivors for research. More rigorous research in this area is necessary.

Secondly, there were limitations related to the review: While every effort was made to include as many studies as possible, the term community health worker represents a very broad concept with many different terms used to describe CHWs in different settings. It is possible that some of these terms were not included in our search strategy. We also included studies that did not define their volunteers as CHWs or volunteers who did not fit the typical WHO definition (for instance belonging to and being selected by the community) as long as they delivered similar services to CHWs. Additionally, our search terms for sexual violence did not include IPV. While we are confident that our search would have picked majority of the IPV studies reporting sexual IPV as an outcome, it is possible that we missed some studies reporting sexual IPV that was not disaggregated from 
other forms of IPV. Similarly, children were poorly represented among the studies reviewed. It is therefore possible that our findings particularly on the acceptability and feasibility of CHWs may differ among survivors of IPV and children.

Despite the limitations of the current evidence base, a number of important findings emerged. Some of the reviewed studies reported that CHWs were trusted members of the community and this trust can enhance their role in awareness raising and mobilisation. This trusted position has enabled CHWs to successfully provide services for other health conditions such as maternal and child health, HIV, TB, malaria, and mental health [28]. Trust is likely to be of key importance for sexual violence, a stigmatised condition; however as our review highlighted, it is also possible that because of stigma and socio-cultural norms, people are reluctant to seek treatment or CHW do not recognise abuse [48]. This echoes findings of a recent systematic review which found that contextual elements such as sociocultural factors influenced CHWs performance [54]. While CHWs have been shown to be beneficial in improving uptake of care, quality of life and retention in care for other socially stigmatising conditions such as HIV [29], a study in Uganda found that due to a desire to keep their condition confidential, people living with HIV preferred CHWs who were from a different village [55].

Although not explored in any studies in this review, CHWs could play an important role in increasing access and adherence to HIV post-exposure prophylaxis (HIV PEP) as well as other treatment for survivors such as emergency contraceptive. CHWs have been used to deliver antiretroviral treatment in Uganda and Kenya for HIV-positive people at home thus making treatment more accessible and affordable [38]. In Madagascar, CHWs routinely provide contraceptives and can effectively perform pregnancy tests to inform their decision on contraceptive prescription [56]. Furthermore, a study in South Africa found that providing proactive follow up with flexible follow up locations such as the survivor's home achieved high follow up and HIV PEP completion rates [23]. However, the authors caution that this intervention involved investing substantial resources, particularly the use of trained nurses. Community health workers, with adequate training and clarity of roles, could be a viable substitute to trained nurses in providing this type of proactive follow up in resourceconstrained settings.

Our review found that only three of the seven studies included information on socio-demographic characteristics of CHWs and none included information on how these factors may have contributed to outcomes. A systematic review on factors influencing CHWs' performance found that CHW' characteristics such as a high education level, experience with health condition, social status and supervision are crucial determinants of CHWs performance [57]. In addition, CHWs' socio-demographic characteristics such as age, gender and marital status may influence clients' perception of CHWs' performance of specific tasks, quality of service and likelihood of dropping out. Given the highly gendered nature of sexual violence, these factors are likely to be relevant not only in terms of performance but also acceptability of CHWs services to survivors. Future studies should not only explore the effect of these sociodemographic characteristics on the acceptability of CHWs to survivors but also their effect on outcomes such as stigma, CHWs' attitudes, CHWs' willingness to offer sexual violence services and CHWs' accessibility and availability.

The only programme that reported ease of delivery and minimal costs in programme had very specialised volunteers delivering a specific training intervention to a very small group. This is unlike typical CHWs programmes where $\mathrm{CHWs}$ have multiple responsibilities and often have to dedicate more time to their different roles. Some of the reviewed studies reported challenges that impact on the feasibility of CHWs services to survivors including long travel distances, too many responsibilities, lack of recognition and other commitments. These challenges, along with others identified in various studies, are systemic challenges which are not unique to sexual violence but affect CHWs in general, and need to be addressed to improve effectiveness of CHWs $[29,31,58]$.

Lastly, our review included studies from low and middle-income countries (LMICs) as well as highincome countries. This was necessary given the limited number of studies available and the exploratory nature of our review. Nevertheless, we recognise that the available resources may influence the type of CHWs and the services they offer to survivors in the different settings. Although the information provided could not allow us to critically evaluate the differences in the models of CHWs in high-income countries compared to LMICs there were a few notable differences. The data suggest that CHWs in high-income countries were more likely to be trained for a specific intervention, attached to a specific sexual violence programme and responsible for fewer survivors $[47,50,51]$. In contrast, CHWs from LMICs were more likely to be general CHWs with sexual violence services as just one of the many healthcare services they provided [49, 52]. Thus, appropriate models of CHWs may differ depending on the resources available and more research is needed to establish the models that work for specific contexts.

\section{Implications and recommendations for future research}

In line with current roles of CHWs, activities that CHWs could be involved in span the whole spectrum of care 
from primary to tertiary prevention with CHWs carrying out activities to reduce stigma and increase social support at the community level, supportive counselling, providing linkages and referral to services, supporting adherence to treatment and retention in care. A major challenge in the current review was lack of uniformity and measureable outcomes to allow comparability across different studies. Studies need to explore further various indicators of acceptability and feasibility including willingness to use, satisfaction with services, ease of delivery, quality and uptake of services, availability of resources, adequacy of training and cost-effectiveness of services. Measureable outcomes that could be assessed in studies evaluating the performance of CHWs for sexual violence care could include proportion of survivors receiving treatment within the recommended $72 \mathrm{~h}$, completion of the 28 days HIV PEP and acute mental health outcomes such anxiety. Longterm outcomes include mental health complications such as depression and post-traumatic stress, STIs e.g. HIV, access to other services e.g. legal and justice, and effects on work or education.

Furthermore, it is important to consider the model of care (community and facility based, or just community), and the type of services to be offered as these will have an impact on the availability of resources and the type of training required. While the current studies reported both models and a variety of services, there was no assessment on the effectiveness of either models or the training and resources required.

Lastly, future studies should assess acceptability, feasibility and specific services for different forms of sexual violence (stranger versus intimate partners/known perpetrators) as well as age (children versus adults) as these may influence the programme design. A notable weakness in the available literature is the limited number of survivors or CHWs interviewed. To design effective CHWs programmes in sexual violence, there is need to engage more survivors and robustly monitor outcomes in CHW-led interventions. Longitudinal studies following up survivors, both with and without $\mathrm{CHWs}$ intervention, with clearly defined measureable outcomes can provide useful comparative data.

\section{Conclusions}

This review points to a potential for CHWs providing support healthcare services for sexual violence but there is lack of quality evidence on appropriate models, acceptability of the services to survivors and feasibility of delivering the services. Improving services for sexual violence survivors through CHWs is only possible if acceptable and feasible models of care can be established. Overall, the studies reviewed were not designed to measure the effectiveness of CHWs services for sexual violence. Further research to establish survivor's views on these services, and the effectiveness of these services, is crucially needed.

\section{Additional file}

Additional file 1: Appendix 1. Database Search Strategy. Appendix 2. Methodological quality rating of quantitative studies. Appendix 3. Methodological quality assessment for qualitative studies. (DOCX 57 kb)

\section{Abbreviations}

AIDS: Acquired Immune Deficiency Syndrome; CHW: Community Health Worker; CSA: Child Sexual Abuse; EPHPP: Effective Public Health Practice Project; HIV: Human Immunodeficiency Virus; NGO: Non-governmental Organisation; NICE: National Institute for Health and Clinical Excellence; PEP: Post Exposure Prophylaxis; PRISMA: Preferred Reporting Items for Systematic reviews and Meta-Analyses; PROSPERO: International Prospective Register of Systematic Reviews; SV: Sexual Violence; TB: Tuberculosis; WHO: World Health Organisation

\section{Acknowledgements}

Not Applicable.

Funding

We acknowledge the funding by the Commonwealth Scholarship Commission for the PhD studentships for AG \& BK.

Availability of data and materials

Data supporting the findings of this study are found in the included tables and Additional file 1.

\section{Authors' contributions}

AG designed the review, developed the inclusion criteria, screened titles and abstracts, appraised the quality of included papers, and drafted the manuscript. KD reviewed the study protocol and inclusion criteria and provided substantial input to the manuscript. JS, MC and IM reviewed the study protocol. BK read and screened articles for inclusion. All authors critically reviewed drafts and approved the final manuscript.

\section{Ethics approval and consent to participate}

This systematic review was part of a bigger study that involved research with human subjects. Ethical approval for the whole study was obtained from the institutional ethics review committee of London School of Hygiene and Tropical Medicine (Ref. 9896) and Kenyatta University (Ref. PKU/386/E32).

Consent for publications

Not applicable.

\section{Competing interests}

The authors declare that they have no competing interests.

\section{Publisher's Note}

Springer Nature remains neutral with regard to jurisdictional claims in published maps and institutional affiliations.

\section{Author details}

${ }^{1}$ Department of Global Health and Development, Faculty of Public Health and Policy, London School of Hygiene and Tropical Medicine, Keppel Street, London WC1E 7HT, UK. ${ }^{2}$ Department of Community Health, School of Public Health, Kenyatta University, Nairobi, Kenya. ${ }^{3}$ Mbarara University of Science \& Technology, Mbarara, Uganda. ${ }^{4}$ Department of Pharmacy, Kent University, Kent, UK.

Received: 9 June 2017 Accepted: 4 October 2017

Published online: 12 October 2017

\section{References}

1. WHO, WHO multi-country study on women's health and domestic violence against women: summary report of initial results on prevalence, health 
outcomes and women's responses. 2005, World Health Organization: Geneva, Switzerland.

2. Devries KM, et al. The global prevalence of intimate partner violence against women. Science. 2013;340(6140):1527-8.

3. Abrahams N, et al. Worldwide prevalence of non-partner sexual violence: a systematic review. Lancet. 2014:383

4. WHO, Global and regional estimates of violence against women: prevalence and health effects of intimate partner violence and non-partner sexual violence. 2013, World Health Organization: Geneva, Switzerland.

5. Bebbington $\mathrm{P}$, et al. Childhood sexual abuse and psychosis: data from a cross-sectional national psychiatric survey in England. Br J Psychiatry. 2011; 199(1):29-37.

6. Young TK, Katz A. Survivors of sexual abuse: clinical, lifestyle and reproductive consequences.[erratum appears in CMAJ 1998 Sep 22;159(6): 650]. Can Med Assoc J. 1998;159(4):329-34.

7. Bebbington PE, et al. Suicide attempts, gender, and sexual abuse: data from the 2000 British psychiatric morbidity survey. Am J Psychiatr. 2009;166(10): $1135-40$.

8. Nelson DB, et al. Childhood violence and behavioral effects among urban pregnant women. J Women's Health. 2010;19(6):1177-83.

9. Longombe AO, Claude KM, Ruminjo J. Fistula and traumatic genital injury from sexual violence in a conflict setting in eastern Congo: case studies. Reprod Health Matters. 2008;16(31):132-41.

10. Al Mamun A, et al. Does childhood sexual abuse have an effect on young adults' nicotine disorder (dependence or withdrawal)? Evidence from a birth cohort study. Addiction. 2007;102(4):647-54.

11. Luce H, Schrager S, Gilchrist V. Sexual assault of women. Am Fam Physician. 2010;81(4):489-95.

12. WHO, Clinical management of rape survivors. Developing protocols for use with refugees and internally displace persons. Geneva, Switzerland: World Health Organization/United Nations High Commissioner for Refugees; 2004.

13. WHO. Guidelines for medico-legal care for victims of sexual violence. 2003 [cited 2016 19th June]; Available from: http://www.who.int/violence_injury_ prevention/publications/violence/med_leg_guidelines/en/.

14. WHO. Post-exposure prophylaxis to prevent HIV infection : joint WHO/ILO guidelines on post-exposure prophylaxis (PEP) to prevent HIV infection. 2007 [cited 2016 20th February]; Available from: http://www.who.int/hiv/ pub/guidelines/PEP/en/.

15. WHO. Responding to intimate partner violence and sexual violence against women. WHO clinical and policy guidelines. 2013 [cited 2016 20/06]; Available from: http://www.who.int/reproductivehealth/publications/ violence/9789241548595/en/.

16. Forbes KM, et al. Management of survivors of sexual assault within genitourinary medicine. Int J STD AIDS. 2008;19(7):482-3.

17. Buard $V$, et al. Characteristics, medical management and outcomes of survivors of sexual gender-based violence, Nairobi, Kenya. Public Health Action. 2013;3(2):109-12.

18. Siika AM, et al. The structure and outcomes of a HIV Postexposure prophylaxis program in a high HIV prevalence setup in western Kenya. J Acquir Immune Defic Syndr. 2009;51(1):47-53. https://doi.org/10.1097/QAI. 0b013e318198a96a.

19. Chacko $L$, et al. Adherence to HIV post-exposure prophylaxis in victims of sexual assault: a systematic review and meta-analysis. Sex Transm Infect. 2012;88(5):335-41.

20. Diniz NMF, et al. Women victims of sexual violence: adherence to chemoprevention of HIV. Revista Latino Americana de Enfermagem. 2007;15(1):7-12.

21. Speight $\mathrm{CG}$, et al. Piloting post-exposure prophylaxis in Kenya raises specific concerns for the management of childhood rape. Trans R Soc Trop Med Hyg. 2006;100(1):14-8.

22. Kim, J.C., et al., Comprehensive care and HIV prophylaxis after sexual assault in rural South Africa: the Refentse intervention study. Vol 338. 2009.

23. Roland $\mathrm{ME}$, et al. Preventing human immunodeficiency virus infection among sexual assault survivors in cape town, South Africa: an observational study. AIDS Behav. 2012;16(4):990-8

24. Garcia MT, et al. Postexposure prophylaxis after sexual assaults: a prospective cohort study. Sex Transm Dis. 2005;32(4):214-9.

25. Abrahams N. And R. Jewkes, Barriers to post exposure prophylaxis (PEP) completion after rape: a South African qualitative study. Cult Health Sex, 2010 12(5): p. 471-484.
26. Christofides NJ, et al. Women's experiences of, and preferences for, services after rape in South Africa: interview study. BMJ. 2006;332(7535):209-12.

27. Muganyizi PS, et al. Managing in the contemporary world: rape victims' and supporters' experiences of barriers within the police and the health care system in Tanzania. J Interpers Violence. 2011;26(16):3187-209.

28. WHO, Global Experience of Community Health Workers for Delivery of Health Related Millennium Development Goals: A Systematic Review, Country Case Studies, and Recommendations for Integration into National Health Systems. 2010, World health organization: Geneva, Switzerland.

29. Mwai GW, et al. Role and outcomes of community health workers in HIV care in sub-Saharan Africa: a systematic review. J Int AIDS Soc. 2013;16:18586.

30. WHO, Strengthening the performance of community health workers in primary health care, in Technical Report Series. 1989, World Health Organization Geneva, Switzerland.

31. Uta Lehmann. D.S., Community health workers: What do we know about them?, in Evidence and Information for Policy, Department of Human Resources for Health. Geneva, Switzerland: World Health Organization; 2007.

32. Wouters E, et al. Impact of community-based support services on antiretroviral treatment programme delivery and outcomes in resourcelimited countries: a synthetic review. BMC Health Serv Res. 2012;12:194.

33. Tomlinson M, et al. Goodstart: a cluster randomised effectiveness trial of an integrated, community-based package for maternal and newborn care, with prevention of mother-to-child transmission of HIV in a south African township. Tropical Med Int Health. 2014;19(3):256-66.

34. Uwimana J, et al. Training community care workers to provide comprehensive TB/HIV/PMTCT integrated care in KwaZulu-Natal: lessons learnt. Tropical Med Int Health. 2012;17(4):488-96.

35. Wouters $E$, et al. Impact of baseline health and community support on antiretroviral treatment outcomes in HIV patients in South Africa. AIDS. 2008;22(18):2545-8.

36. Dowrick $\mathrm{C}$, et al. Problem solving treatment and group psychoeducation for depression: multicentre randomised controlled trial. Br Med J. 2000; 321(7274):1450,

37. Ertl V, et al. Community-implemented trauma therapy for former child soldiers in northern Uganda: a randomized controlled trial. J Am Med Assoc. 2011;306(5):503-12.

38. Decroo T, et al. Community-based antiretroviral therapy programs can overcome barriers to retention of patients and decongest health services in sub-Saharan Africa: a systematic review. Int Health. 2013;5(3):169-79.

39. Moher $\mathrm{D}$, et al. Preferred reporting items for systematic reviews and metaanalyses: the PRISMA statement. PLoS Med. 2009;6(7):e1000097.

40. Etienne K, et al. World report on violence and health. Geneva, Switzerland: World Health Organization; 2002.

41. Noyes J, L.S., Extracting qualitative evidence., in Supplementary Guidance for Inclusion of Qualitative Research in Cochrane Systematic Reviews of Interventions, B.A. Noyes J, Hannes K, harden a, Harris J, Lewin S, Lockwood C editor. 2011, Cochrane Collaboration Qualitative Methods Group.

42. EPHPP. Quality Assessment Tool for Quantitative Studies. 1998 [cited 2016 February]; Available from: http://www.ephpp.ca/tools.html.

43. Wong WC, Cheung CS, Hart GJ. Development of a quality assessment tool for systematic reviews of observational studies (QATSO) of HIV prevalence in men having sex with men and associated risk behaviours. Emerg Themes Epidemiol. 2008:5(1):1-4.

44. Higgins JPT and G. S, Cochrane Handbook for Systematic Reviews of Interventions. 2011: The Cochrane Collaboration.

45. NICE, The guidelines manual (Appendices) Appendix 1 Methodology checklist: qualitative studies. 2009. p. 208-217.

46. Gale NK, et al. Using the framework method for the analysis of qualitative data in multi-disciplinary health research. BMC Med Res Methodol. 2013; 13(1):1-8.

47. Barron IG, Topping KJ. Survivor experience of a child sexual abuse prevention program: a pilot study. J Interpers Violence. 2013;28(14):2797-812.

48. Itzhaky H, York AS. Child sexual abuse and incest: community-based intervention. Child Abuse Negl. 2001;25(7):959-72.

49. Anjalee Kohli, et al. A Congolese community-based health program for survivors of sexual violence. Conflict and Health 2012. 2012;6(6):1-9.

50. Merkin L, Smith MJ. A community based model providing services for deaf and deaf-blind victims of sexual assault and domestic violence. Sexuality \& Disability. 1995;13(2):97-106.

51. Rossman L, Dunnuck C. A community sexual assault program based in an urban YWCA: the grand rapids experience. J Emerg Nurs. 1999;25(5):424-7. 
52. Tanabe $\mathrm{M}$, et al. Piloting community-based medical care for survivors of sexual assault in conflict-affected karen state of eastern Burma. Confl Heal. 2013;7(12)

53. Zraly M, Rubin-Smith J, Betancourt T. Primary mental health care for survivors of collective sexual violence in Rwanda. Global Public Health. 2011; 6(3):257-70.

54. Kok MC, et al. How does context influence performance of community health workers in low- and middle-income countries? Evidence from the literature. Health Res Policy Syst. 2015;13(1):1-14.

55. Alamo S, et al. Task-shifting to community health workers: evaluation of the performance of a peer-led model in an antiretroviral program in Uganda. AIDS Patient Care STDs. 2012;26(2):101-7.

56. Comfort AB, et al. Providing free pregnancy test kits to community health workers increases distribution of contraceptives: results from an impact evaluation in Madagascar. Contraception. 2016;93(1):44-51.

57. Kok MC, et al. Which intervention design factors influence performance of community health workers in low- and middle-income countries? A systematic review. Health Policy Plan. 2014:1-27.

58. Tulenko $\mathrm{K}$, et al. Community health workers for universal health-care coverage: from fragmentation to synergy. Bull World Health Organ. 2013; 91(11):847-52

Submit your next manuscript to BioMed Central and we will help you at every step:

- We accept pre-submission inquiries

- Our selector tool helps you to find the most relevant journal

- We provide round the clock customer support

- Convenient online submission

- Thorough peer review

- Inclusion in PubMed and all major indexing services

- Maximum visibility for your research

Submit your manuscript at www.biomedcentral.com/submit
C Biomed Central 